\title{
Rehabilitation of Advanced Cancer Patients in Palliative Care Unit
}

\author{
Chang Hyun Lee, MD ${ }^{1}$, Jong Kyu Kim, MD', Hyun Jung Jun, MD, PhD², \\ Duk-Joo Lee, $\mathrm{MD}^{2}$, Wook Namkoong, $\mathrm{MD}^{3}$, Jae $\mathrm{Ho} \mathrm{Oh}, \mathrm{MD}^{1}$
}

${ }^{1}$ Department of Physical Medicine and Rehabilitation, ${ }^{2}$ Division of Medical Oncology and Hematology, Department of Internal Medicine, ${ }^{3}$ Department of Family Medicine, Seoul Medical Center, Seoul, Korea

Objective To evaluate the compliance and satisfaction of rehabilitation recommendations for advanced cancer patients hospitalized in the palliative care unit.

Methods Advanced cancer patients admitted to a hospice palliative care unit were recruited. Patients with advanced cancer and a life expectancy of less than 6 months, as assumed by the oncologist were included. Patients who were expected to die within 3 days were excluded. ECOG and Karnofsky performance scales, function ambulatory category, level of ambulation, and survival days were evaluated under the perspective of comprehensive rehabilitation. Problem-based rehabilitations were provided categorized as physical therapy at the gym, bedside physical therapy, physical modalities, medications and pain intervention. Investigation of compliance for each category was completed. Patient satisfaction was surveyed using a questionnaire.

Results Forty-five patients were recruited and received evaluations for rehabilitation perspective. The subjects were reported to have gait-related difficulties $(71.1 \%)$, pain $(68.9 \%)$, poor medical conditions (68.9\%), bladder or bowel problems (44.4\%), dysphagias (11.1\%), mental status issues (11.1\%), edemas (11.1\%), spasticity (2.2\%), and pressure sores $(2.2 \%)$. In the t-test, patients with good compliance for GymPT showed higher survival days $(\mathrm{p}<0.05)$. In the satisfaction survey, patients with performance scales showed a greater satisfaction in Spearman's correlation analysis $(\mathrm{p}<0.05)$.

Conclusion Advanced cancer patients admitted to the hospice palliative care unit have many rehabilitation needs. Patients with a longer survival time showed better compliance for GymPT. Patients with a better performance scale showed a higher satisfaction. Comprehensive rehabilitation may be needed to advanced cancer patients in the hospice palliative care unit.

Keywords Neoplasms, Rehabilitation, Palliative care, Hospice care

Received May 19, 2017; Accepted July 13, 2017

Corresponding author: Jong Kyu Kim

Department of Physical Medicine \& Rehabilitation, Seoul Medical Center, 156 Sinnae-ro, Jungnang-gu, Seoul 02053, Korea. Tel: +82-2-2276-7474, Fax: +82-2-2276-8534, E-mail: jongkyukim@seoulmc.or.kr

ORCID: Chang Hyun Lee (http://orcid.org/0000-0003-0501-3663); Jong Kyu Kim (http://orcid.org/0000-0003-1441-9227); Hyun Jung Jun (http://orcid. org/0000-0003-0825-8068); Duk-Joo Lee (http://orcid.org/0000-0003-1220-322X); Wook Namkoong (http://orcid.org/0000-0003-0181-309X); Jae Ho Oh (http://orcid.org/0000-0002-2203-897X).

() This is an open-access article distributed under the terms of the Creative Commons Attribution Non-Commercial License (http://creativecommons.org/ licenses/by-nc/4.0) which permits unrestricted noncommercial use, distribution, and reproduction in any medium, provided the original work is properly cited. Copyright $\odot 2018$ by Korean Academy of Rehabilitation Medicine 


\section{INTRODUCTION}

In Korea, cancer accounts for the maximum number of deaths, and is increasing [1]. At the same time, the prevalence of cancer and cancer survivorship is also increasing. Cancer rehabilitation is becoming increasingly important in various categories to improve the quality of life (QoL) in cancer patients [2].

Beyond the cancer survivors, patients who are currently receiving treatment also have QoL issues and require rehabilitation. Rehabilitation is important in all phases of cancer treatment, starting from before surgery to the terminal stage [3]. Exercise provides benefits to reduce fatigue during adjuvant radiotherapy in breast cancer patients [4]. Incurable cancer patients could have a relatively long lifetime, and they experience various burdens from cancer and QoL issues $[5,6]$. Rehabilitation can help these patients.

Patients who receive palliative care show many QoLrelated problems and activities of daily living (ADL) limitations. For those in hospice palliative care, maintaining QoL and human dignity is imperative [7-9]. However, many palliative care physicians place greater emphasis on pain management so that various ADL problems can be neglected. Even though patients may die soon, they have the right to maintain their dignity, and a rehabilitation approach can improve QoL and independent ADL. Research conducted on exercises in palliative care chronicled the benefits on QoL, fatigue, muscle mass, strength, and treatment compliance [10]. Another study reported that rehabilitation gym circuits classes in a hospice center improve a gait function [11]. However, approaches with comprehensive rehabilitative perspectives have not been studied.

Rehabilitation medicine is oriented toward physical recovery, removal of a functional barrier, and social reintegration. Thus, many physicians including physiatrists have no interest in patients with progressive diseases or their course of death. However, many patients with advanced cancer suffer from impaired ADL or a low QoL $[6,12]$. If a rehabilitation approach can be helpful, it should be used for these patients. Rehabilitation medicine improves human dignity and will be effective for patients who are near to death.

To our knowledge, no study has analyzed active rehabilitation approaches targeting terminal stage cancer pa- tients admitted to hospice palliative care. We evaluated advanced cancer patients in a hospice palliative care unit with rehabilitation perspectives, recommended rehabilitation interventions, and investigated the compliance and satisfaction of the patients.

\section{MATERIALS AND METHODS}

\section{Participants}

From June 2015 to January 2016, patients admitted to the Hospice \& Palliative Care Unit of Seoul Medical Center were primarily recruited. Admission criteria to the palliative care unit included diverse terminal stage cancer with a life expectancy of less than 6 months based on the opinion of two oncologists. Patients who were expected to die within 3 days were excluded. Except for those individuals, all patients were informed of this study by the oncologists. The oncologists received informed consent from the patients. If the patients could not communicate due to their condition, we received permission from the primary caregiver. The physiatrist then visited the patients and explained the rehabilitation approaches, including the survey, evaluation, and rehabilitation program. If the patients or caregivers did not want to get involved in this program, they were excluded.

We investigated the basic characteristics, initial pain scores using the visual analog scale, Eastern Cooperative Oncology Group (ECOG) performance scale, and the Karnofsky performance scale. ECOG and Karnofsky performance scales are widely used functional scales that describe the functional ability of cancer patients. ECOG ranges from 0 to 5 , where 0 means fully active, and 5 means patient death [13]. The Karnofsky scale ranges from 0 to 100, where 0 indicates the dead and 100 indicates the normal [14]. One week after the initial survey, we followed-up with the cancer survivors. Follow-up surveys were completed in the 1st and 2nd week. After 6 months of closing this study, we investigated the survival days from the initial evaluation day using medical records.

\section{Rehabilitation}

We evaluated the patients regarding rehabilitation comprehension of their problems and functions. We made a problem list from a rehabilitation viewpoint, and for each problem, we recommended the rehabilitative approach. 
We evaluated their function of self-care ADL (eating, grooming, dressing, using a toilet, bathing, sphincter control, and ambulation). We also evaluation ambulation level using the Functional Ambulatory Category (FAC) [15]. FAC cannot represent wheelchair ambulators. Thus, we created and checked a 5-scale ambulation ability tool, level of ambulation. Level of ambulation consisted of five categories: (1) walking independently, (2) walking with supervision or minimal assistance, (3) walking with moderate to maximal assistance, (4) propelling the wheelchair manually, and (5) totally dependent. Level of ambulation was checked under a level surface, for $10-\mathrm{m}$ ambulation with or without holding a monocane or pole.

According to the patient's problems and needs, we recommended rehabilitation approaches, such as physical therapy at the gym (GymPT), bedside physical therapy (bedsidePT), physical modalities to control pain, medication recommendations, a caregiving method change, and pain intervention. GymPT included standing training using a tilt table, parallel bar walking, stationary bicycle riding, balance, and endurance exercise, and was recommended to the patients with partial ambulation ability and stable vital signs. If the patients had unstable vital signs or were unable to ambulate to the gym, BedsidePT was recommended, which consisted of passive and active range of motion exercises. Physical modalities were recommended to those who had localized pain, and they consisted of transcutaneous electrical stimulation and heat therapy. Medication recommendations consisted of all kind of medications affecting QoL, such as pain-killers, neuropathic pain drugs, and medications aiding bowel movements or voiding. Changing methods consisted of recommendations of feeding methods, sore dressing, bladder and bowel care, and exercise methods given by caregivers. The rehabilitation intervention treatment was agreed to by the patient or the caregiver.

\section{Compliance and satisfaction}

For the five rehabilitation approaches (GymPT, bedsidePT, physical modalities, medication, and pain intervention), we monitored the compliance of each item. We classified the compliant group as the patients who followed our rehabilitation recommendations. The others were classified as the non-compliant group.

We also received satisfaction-related questionnaires from the patients who participated in rehabilitation in- tervention for more than a week and agreed to respond. If the patients could not reply, the primary caregiver could answer. We use a general satisfaction score with the 11-point Likert scale (0-10). We also monitored five satisfaction items (pain relief, maintenance of ADL, improvement of fatigue, improvement of psychologic symptoms, and preference to receive any treatment) and five dissatisfaction items (no improvement in any symptom, no improvement in activity of daily living, too hard to take rehabilitation treatment, aggravation of fatigue, and aggravation of psychologic symptoms). We counted satisfaction scores (each +1 point) and dissatisfaction score (each +1 point) for each item. The total satisfaction score was calculated as the satisfaction score minus the dissatisfaction score.

\section{Statistics}

Two-tailed independent t-tests were used to compare means between the compliant and non-compliant groups for each rehabilitation interventions. A Spearman's correlation was used for patient characteristics and satisfaction scores. All statistical analyses were performed using SPSS version 20.0 (IBM SPSS Inc., Armonk, NY, USA). A $\mathrm{p}<0.05$ was considered statistically significant.

This study was approved by the Institutional Review Board of Seoul Medical Center (No. 2015-046).

\section{RESULTS}

\section{Patients}

A total of 62 patients were initially included during the

Table 1. Demographic features $(n=45)$

\begin{tabular}{lc}
\hline \multicolumn{1}{c}{ Characteristic } & Value \\
\hline Age (yr) & $64.0 \pm 13.4$ \\
\hline Sex & \\
\hline Male & 19 \\
\hline Femal & 26 \\
\hline Survival days & $40.1 \pm 38.2$ \\
\hline Median & 30 \\
\hline ECOG performance scale & $2.97 \pm 2.10$ \\
\hline Karnofsky performance scale & $50.44 \pm 16.92$ \\
\hline Functional Ambulation Category & $2.10 \pm 0.67$ \\
\hline
\end{tabular}

Values are presented as mean \pm standard deviation. ECOG, Eastern Cooperative Oncology Group. 
study period. After the explanation about the rehabilitation program with the physiatrist, 45 patients (19 male and 26 female patients) agreed to undertake the rehabilitation approach. The mean age of the subjects was 64 years. The median number of survival days was 30 . Demographic characteristics and functional levels are described in Table 1 . The sites of the primary cancer were classified into 10 sites, and participants showed various functional status, FAC, and levels of ambulation (Fig. 1).

\section{Problems}

We investigated both subjective and objective prob- lems. Subjective problems were complaints from patients or caregivers, which were not evaluated by any diagnostic methods. They consisted of gait-related difficulty, pain, spasticity (spastic feeling), dysphagia (feeling of swallowing difficulty), and bladder or bowel management problems. Spasticity was not evaluated objectively. Dysphagia also depended on patients' or caregivers' appeal. Bladder problems consisted of incontinence, urination frequency, urgency, dysuria, and the need for a device for voiding. Bowel problems were constipation, diarrhea, and incontinence. Thirty-two patients $(71.1 \%)$ complained of gait-related difficulty (Table 2). Objective problems were
A Site of primary cancer

$$
\text { Lung }
$$

Gallbladder and bile duct

$$
\text { Pancreas }
$$

Colon and rectum

$$
\text { Brain }
$$

Liver

Breast

Uterus

Others $^{\mathrm{a})}$

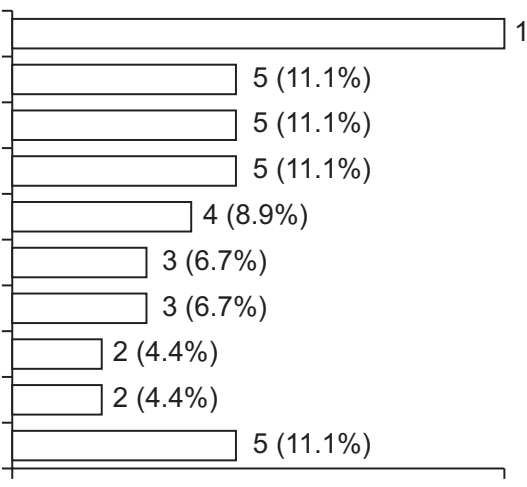

(B) ECOG performance scale $11(24.4 \%)$

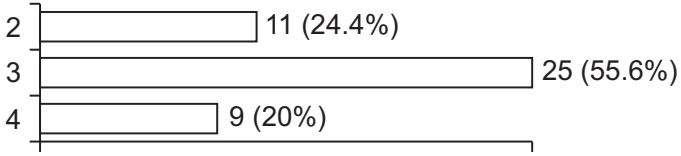

(C) Karnofsky performance scale

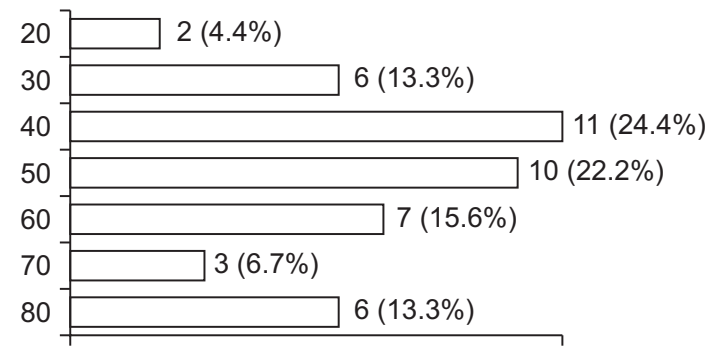

D Functional ambulatory category

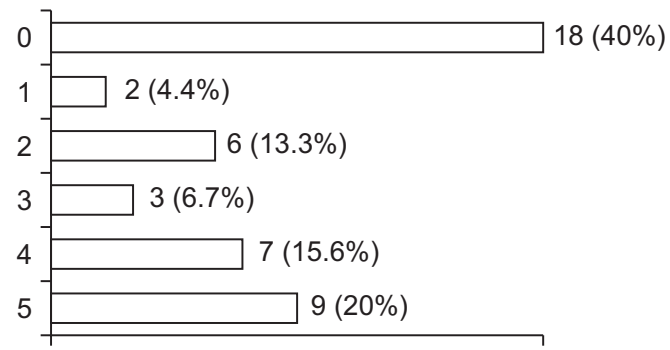

(E)

Level of ambulation

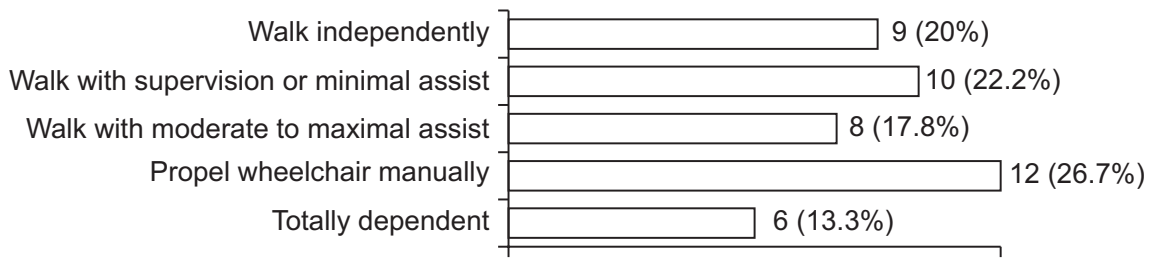

Fig. 1. Characteristics of included patients. (A) Site of primary cancer, (B) Eastern Cooperative Oncology Group (ECOG) performance scale, (C) Karnofsky performance scale, (D) Functional Ambulatory Category, and (E) level of ambulation. Others include ovary, esophagus, prostate, bone (osteosarcoma) and blood (leukemia). 
Table 2. Results of problems survey

\begin{tabular}{|cc}
\hline & No. (\%) \\
\hline Subjective problems ${ }^{\text {a) }}$ & \\
\hline Gait-related difficulty & $32(71.1)$ \\
\hline Pain & $1(68.9)$ \\
\hline Spasticity & $1(2.2)$ \\
\hline Dysphagia & $5(11.1)$ \\
\hline Bladder/bowel management problem & $20(44.4)$ \\
\hline Objective problems ${ }^{\text {b) }}$ & \\
\hline Not alert mental status & $5(11.1)$ \\
\hline Pressure sore & $1(2.2)$ \\
\hline Edema & $5(11.1)$ \\
\hline Poor medical conditions & $31(68.9)$ \\
\hline Pain subtypes ${ }^{\text {c) }}$ & \\
\hline Cancer-related pain & $11(24.4)$ \\
\hline Musculoskeletal pain & $21(46.7)$ \\
\hline Neuropathic pain & $1(2.2)$ \\
\hline CRPS pain & $1(2.2)$ \\
\hline
\end{tabular}

${ }^{\text {a) }}$ Subjective problems appealed by the patients or patients' care-givers.

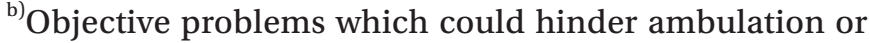
physical therapy for patients.

${ }^{c}$ Pain subtypes was decided by the physiatrist on the basis of physical exams, vital signs, laboratory data and medical records.

problems identified or diagnosed by the physiatrist or other medical personnel. They included not-alert mental status, pressure sore, edema, sensory dysfunction, and poor medical conditions. Not alert mental state consisted of coma, semi-coma, stuporous, and delirious states. A pressure sore was defined above National Pressure Ulcer Advisory Panel stage 2. Edema included any type of limb edema. Sensory dysfunction indicated a reduced sensory function that increased the risk of injury. Poor medical conditions included any barriers of performing exercises at the gym, such as low hemoglobin/hematocrit, unstable vital signs, bleeding tendency, fever, and evidence of acute infection or inflammation. Thirty-one patients (68.9\%) exhibited poor medical conditions (Table 2).

Pain was classified into cancer-related pain, musculoskeletal pain, neuropathic pain, and Complex Regional Pain Syndrome (CRPS) pain. Cancer-related pain was defined as pain near the primary cancer site, pain near the direct invasion or distant metastasis site, post-operative pain at the operation site, and radiotherapy related pain.

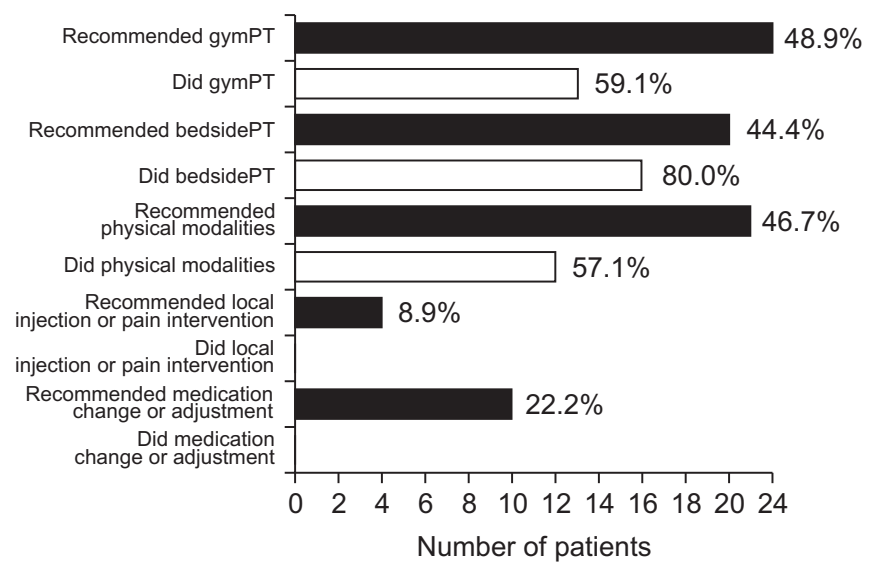

Fig. 2. Compliance of recommended rehabilitation approach.

Musculoskeletal pain included low back pain, radicular pain, frozen shoulder, shoulder impingement associated pain, joint pain irrelevant to the cancer site, osteoporosis induced compression fracture, and myofascial pain syndrome. CRPS pain included type 1 or type 2 CRPS. Musculoskeletal pain was the most frequent complaint, with 21 patients (46.7\%) complaining of this type of pain. However, 11 patients $(24.4 \%$ ) showed cancer-related pain (Table 2).

\section{Rehabilitation}

According to each patient's problem, we recommended GymPT, bedsidePT, physical modalities, medication change, and pain interventions. GymPT included exercises at the gym, such as tilted table standing, stander, motormed, stationary bike, parallel bar gait, and any other gait training. BedsidePT consisted of active and passive range or motion exercise at the bedside. Physical modalities included heat therapy, such as a hot pack and ultrasound, transcutaneous electrical nerve stimulation or interferential current therapy, intermittent pneumatic compression, and manual lymphatic drainage. Medication change indicated a change or addition of pain medications, gastrointestinal drugs, bladder or bowel management medications, and edema control medications. Pain interventions consisted of musculoskeletal interventions including steroid injection, peripheral nerve blocks, and epidural steroid injections.

\section{Compliance}

Of GymPT-recommended 22 patients, only 13 com- 
Table 3. Compliance to rehabilitation approach

\begin{tabular}{llcclcc}
\hline & & Age $(\mathbf{y r})$ & Survival days & FAC & ECOG PS & Karnofsky PS \\
\hline GymPT $^{\text {a) }}$ & Yes & 67.4 & $46.6^{*}$ & 3.1 & 2.6 & 60.0 \\
& No & 61.0 & $23.7^{*}$ & 3.6 & 2.4 & 63.3 \\
\hline BedsidePT $^{\text {b) }}$ & p-value & 0.243 & 0.13 & 0.575 & 0.521 & 0.625 \\
& Yes & 66.6 & 45.3 & 0.4 & 3.3 & 38.8 \\
\hline \multirow{2}{*}{ Physical modalities $^{\text {c) }}$} & No & 58.3 & 60.8 & 1.0 & 3.3 & 45.0 \\
& p-value & 0.268 & 0.787 & 0.617 & 0.819 & 0.333 \\
& Yes & 63.3 & 37.9 & 3.3 & 2.6 & 56.7 \\
& No & 58.5 & 27.9 & 1.8 & 2.9 & 55.0 \\
\hline & p-value & 0.511 & 0.32 & 0.14 & 0.398 & 0.845 \\
\hline
\end{tabular}

FAC, Functional Ambulatory Category; ECOG PS, Eastern Cooperative Oncology Group; PS, performance scale.

${ }^{\text {a) }}$ Patients followed rehabilitation recommendation for physical therapy at Gym.

${ }^{b}$ Patients followed rehabilitation recommendation for physical therapy at bedside.

${ }^{c)}$ Patients followed rehabilitation recommendation for physical modalities.

${ }^{*} \mathrm{p}<0.05$.

pleted their rehabilitation. However, $80 \%$ patients agreed for bedsidePT. Although physical modalities were approved for 21 patients, only 12 received the treatment. No one took the recommendation for pain intervention and medication change item. Compliance of each rehabilitation recommendation is described in Fig. 2. For GymPT, a significant difference was observed in the survival days between the compliant and non-compliant groups. Compliant to GymPT patients showed higher survival (Table 3).

\section{Satisfaction}

Only 13 patients completed the satisfaction-dissatisfaction questionnaire. They scored an overall satisfaction of 6.69 out of 10 (Table 4). Patients with a higher satisfaction score showed better ECOG and Karnofsky performance scales with significant correlations in Spearman correlation (Table 5).

\section{DISCUSSION}

Cancer rehabilitation has emerged as a reasonable option for cancer survivors. Nowadays, cancer rehabilitation is important at various stages of cancer patients, from initial treatment until death $[12,16,17]$. Many advanced cancer patients have a life expectancy of more than 12 months. During this period, they have various QoL concerns [6]. In addition, cancer patients receiving palliative care need rehabilitative approaches for various reasons $[3,10]$. Benefits of exercises are well known in
Table 4. Result of satisfaction and dissatisfaction questionnaire

\begin{tabular}{ll}
\hline \multicolumn{1}{c}{ Item } & Value $^{\mathrm{a})}$ \\
\hline Satisfaction & \\
Pain relief & $4(30.8)$ \\
\hline Maintenance of activity of daily living & $1(7.7)$ \\
\hline Improvement from feeling of fatigue & $2(15.4)$ \\
\hline Improvement of psychologic symptom & $3(23.1)$ \\
\hline To prefer receiving any treatment & $7(53.8)$ \\
Dissatisfaction & \\
No improvement in any symptom & $0(0)$ \\
\hline No improvement in activity of daily living & $0(0)$ \\
Treatment is too hard to carry on & $1(7.7)$ \\
\hline Aggravation for feeling of fatigue & $0(0)$ \\
\hline Aggravation of psychologic symptom & $1(7.7)$ \\
\hline Overall satisfaction $(0-10)^{\mathrm{b})}$ & $6.69 \pm 3.17(7)$ \\
\hline Satisfaction score $(0-5)^{\mathrm{c})}$ & $1.31 \pm 0.63(1)$ \\
Dissatisfaction score $(0-5)^{\mathrm{d})}$ & $0.15 \pm 0.38(0)$ \\
\hline
\end{tabular}

Values are presented as number (\%) or mean \pm standard deviation (median).

a) Total patients were 13 who answered the survey by himself or by his/her care-giver.

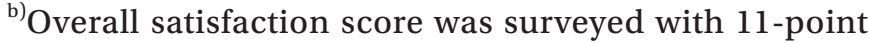
Likert scale (0-10).

${ }^{c, d)}$ Satisfaction and dissatisfaction score were calculated as sum of number which answered as positive within satisfaction/dissatisfaction items, each item scores 1 point. 
Table 5. Correlations between patient characteristics and satisfaction

\begin{tabular}{llcccc}
\hline & General satisfaction & $\begin{array}{c}\text { Satisfaction } \\
\text { score }^{\mathbf{b})}\end{array}$ & $\begin{array}{c}\text { Dissatisfaction } \\
\text { score }^{\mathbf{c})}\end{array}$ & $\begin{array}{c}\text { Total satisfaction } \\
\text { score }^{\mathbf{d})}\end{array}$ \\
\hline Age (yr) & Spearman's rho & 0.42 & 0.19 & 0.06 & -0.05 \\
& p-value & 0.149 & 0.952 & 0.853 & 0.874 \\
Survival days & Spearman's rho & 0.01 & 0.16 & -0.23 & 0.26 \\
& p-value & 0.982 & 0.6 & 0.453 & 0.393 \\
FAC & Spearman's rho & 0.25 & 0.33 & -0.03 & 0.31 \\
\multirow{2}{*}{ ECOG PS } & p-value & 0.41 & 0.275 & 0.922 & 0.304 \\
& Spearman's rho & -0.32 & $-0.667^{*}$ & 0.0 & $-0.636^{*}$ \\
Karnofsky PS & p-value & 0.284 & 0.013 & 1.0 & 0.019 \\
& Spearman's rho & 0.01 & $0.62^{*}$ & 0.23 & 0.44 \\
& p-value & 0.97 & 0.025 & 0.445 & 0.137 \\
\hline
\end{tabular}

FAC, Functional Ambulatory Category; ECOG PS, Eastern Cooperative Oncology Group; PS, performance status.

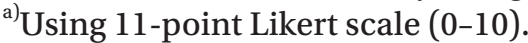

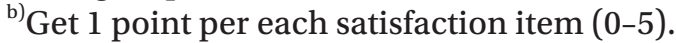

${ }^{c)}$ Get 1 point per each dissatisfaction item (0-5).

${ }^{\mathrm{d})}$ Satisfaction score minus dissatisfaction score.

${ }^{*} \mathrm{p}<0.05$.

various cancer patients as are exercise guidelines for cancer patients [7]. However, rehabilitation does not include only exercises. Rehabilitative perspective categorizes the patients' problems as body impairment, functional limitation, and participation restriction [18]. These holistic approaches are necessary for cancer patients and survivors at a terminal stage.

\section{Ambulation}

Even patients in the terminal stage express strong wishes such as "I want to walk" and "I want to go to the bathroom" [3]. In our study, many patients and caregivers thought walking to the bathroom was very important. In other words, even in hospice, patients and their family wanted to experience meaningful care. Active exercises, including GymPT and bedsidePT may enhance selfesteem and psychological symptoms. Physical modalities do not include any exercise, but to undergo the modalities, patients must go to the therapy room. This may also be helpful to the patients' overall satisfaction. In our opinion, preserving ambulatory function is the largest need that is closely related to self-esteem and QoL in palliative care patients [19]. In hospice care, physiatrists must consider the various needs for ambulation.

Pain

Our investigation revealed that musculoskeletal pain was the most frequently-mentioned complaint. For breast cancer patients, diverse musculoskeletal disorders can provoke shoulder problems [20,21]. Many cancer patients suffer from cancer-related pain and other musculoskeletal pain [22]. This is also applicable to advanced cancer patients receiving palliative care. Proper management of musculoskeletal pain like physical modalities, painkillers, injection therapy, and exercises could reduce pain and opioid use to enhance physical activity and QoL. Among the musculoskeletal pain, frozen shoulders, low back pain, and radicular pain are the most common areas that patients mention. This pain is not fatal, but hinders activity and ADL. It may also aggravate psychological symptoms. Injection therapies such as intraarticular steroid or epidural steroid injections can reduce these kinds of pain immediately, and can help to reduce activity limitation. We suggest that active pain intervention can mitigate these losses.

Frozen shoulders might be due to immobilization and surgery $[20,21]$. Neuropathic pain might be related to chemotherapy $[20,23]$. There was one case of complex regional pain syndrome that was related to brain metastasis. We also observed one case of spastic pain due to spinal cord compression. This disease entity is related to rehabilitation. A rehabilitation approach could afford a proper diagnosis and management for these individuals. Considering the diverse cause of pain and activity limita- 
tions of advanced cancer patients, from an early stage of treatment till their death, a rehabilitative approach can help the patients maintain a higher QoL [17].

\section{Compliance}

For the rehabilitation recommendation, patients with better performance scales showed greater compliance. In GymPT, more compliant patients had longer survival days. The rehabilitative approach consisted of GymPT, bedsidePT, physical modalities, recommendation of pain interventions, and medications. All patients who were recommended for pain interventions and medication change refused the modifications. Almost all patients and caregivers thought they were terminal and did not want any invasiveness. For a similar reason, some patients and care-givers thought that exercises were meaningless. We recommended to some patients changing medications, musculoskeletal pain interventions and different methods of caregiving at bedside, to the patients, but no one participated. Some patients felt that exercise was too hard. We, along with our physical therapists, had little experience with terminal cancer patients. To improve compliance, research regarding the proper intensity of rehabilitation would be necessary. In the satisfaction-related survey, many patients expressed being satisfied with the receipt of any treatment. Hospice care does not equate to doing nothing. We could not perform a study of effectiveness, but patients with better performance showed compliance and satisfaction. It may mean that the rehabilitation approach in patients with better performance could increase their effectiveness. Even though these patients suffer from an incurable disease, early application of comprehensive rehabilitation may be needed.

\section{Limitations}

This study has some limitations. First, this study included patients with various types of cancers. Each cancer may have various characters and burdens to the patients that are diverse. Furthermore, we did not investigate whether cancers were locally advanced or distantly metastatic. Locally advanced cancer may show different features compared with distant metastasis. However, many participants were transferred from other hospitals, little medical information was available on their condition. The patients only wanted to receive palliative care and did not want to undergo more diagnostic tests. A study of a single type of solid cancer will elicit the effectiveness of rehabilitation more precisely. Second, we did not investigate the effectiveness of comprehensive rehabilitation. All the patients in the palliative care unit were near death. As time went by, all patients experienced a functional decline and QoL aggravation. This resulted in poor compliance. Thus, general evaluation to check the effectiveness of rehabilitation was inadequate. We could not find the right tool to monitor the effectiveness. Only the compliance and satisfaction could have been evaluated, and we could not have investigated factors affecting compliance. Furthermore, the satisfaction-related survey was our innovative idea without validation data. We could not find an adequate tool to evaluate the compliance and satisfaction of cancer patients in the terminal stage. For a similar reason, we could not assign a control group. To prove the effectiveness of any intervention, a randomized controlled study is the most powerful. However, for these patients, a controlled study was difficult due to the physical status and some existing ethical issues. Lastly, the follow-up time was short. In our institution, hospitalization in the hospice palliative care unit was limited to 6 weeks (Nowadays, the limitation is 2 months due to the change in insurance policy). The median number of survival days was 30 , and many patients did not complete the 3-week follow-up. Only 11 patients could answer the satisfactionrelated questionnaire. In Korea, many hospice palliative care centers receive patients in the terminal stages of cancer. If a patient with advanced cancer is in the terminal stage, early palliative care and rehabilitation could be helpful to preserve QoL and independent ADL until the end of life. Thus, the development of early palliative care and an early rehabilitation program is necessary. In addition, early palliative rehabilitation research may solve the problems associated with the randomized controlled study. This was a preliminary study and the first study on palliative rehabilitation. A multicenter research study of a larger size and longer duration is needed.

In conclusion, advanced cancer patients hospitalized in hospice palliative care unit face several issues, and comprehensive rehabilitation approaches help to resolve these issues. Individuals with better performance showed more compliance and satisfaction. To improve the palliative rehabilitation program, a larger population is needed. 


\section{CONFLICT OF INTEREST}

No potential conflict of interest relevant to this article was reported.

\section{ACKNOWLEDGMENTS}

This study was supported by a grant of Seoul Medical Center Research Institute (\#15-C17).

\section{REFERENCES}

1. Shin HY, Lee JY, Song J, Lee S, Lee J, Lim B, et al. Cause-of-death statistics in the Republic of Korea, 2014. J Korean Med Assoc 2016;59:221-32.

2. Jo JM, Hwang JH, Lee CH, Kang HJ, Yu JN. The need of cancer patients for rehabilitation services. J Korean Acad Rehabil Med 2010;34:691-700.

3. Okamura H. Importance of rehabilitation in cancer treatment and palliative medicine. Jpn J Clin Oncol 2011;41:733-8.

4. Lipsett A, Barrett S, Haruna F, Mustian K, O'Donovan A. The impact of exercise during adjuvant radiotherapy for breast cancer on fatigue and quality of life: a systematic review and meta-analysis. Breast 2017;32:144-55.

5. Carduff E, Kendall M, Murray SA. Living and dying with metastatic bowel cancer: serial in-depth interviews with patients. Eur J Cancer Care (Engl) 2017.

6. Dasch B, Kalies H, Feddersen B, Ruderer C, Hiddemann W, Bausewein C. Care of cancer patients at the end of life in a German university hospital: a retrospective observational study from 2014. PLoS One 2017;12:e0175124.

7. Hartogh GD. Suffering and dying well: on the proper aim of palliative care. Med Health Care Philos 2017;20:413-24.

8. Kanach FA, Brown LM, Campbell RR. The role of rehabilitation in palliative care services. Am J Phys Med Rehabil 2014;93:342-5.

9. McIlfatrick S, Connolly M, Collins R, Murphy T, Johnston B, Larkin P. Evaluating a dignity care intervention for palliative care in the community setting: community nurses' perspectives. J Clin Nurs 2017;26:4300-12.

10. Eyigor S, Akdeniz S. Is exercise ignored in palliative cancer patients? World J Clin Oncol 2014;5:554-9.

11. Talbot Rice H, Malcolm L, Norman K, Jones A, Lee K, Preston G, et al. An evaluation of the St Christopher's Hospice rehabilitation gym circuits classes: patient uptake, outcomes, and feedback. Prog Palliat Care 2014;22:319-25.

12. Gerber LH. Cancer rehabilitation into the future. Cancer 2001;92(4 Suppl):975-9.

13. Oken MM, Creech RH, Tormey DC, Horton J, Davis TE, McFadden ET, et al. Toxicity and response criteria of the Eastern Cooperative Oncology Group. Am J Clin Oncol 1982;5:649-55.

14. Karnofsky DA, Abelmann WH, Craver LF, Burchenal $\mathrm{JH}$. The use of the nitrogen mustards in the palliative treatment of carcinoma: with particular reference to bronchogenic carcinoma. Cancer 1948;1:634-56.

15. Holden MK, Gill KM, Magliozzi MR, Nathan J, PiehlBaker L. Clinical gait assessment in the neurologically impaired. Reliability and meaningfulness. Phys Ther 1984;64:35-40.

16. DeLisa JA. A history of cancer rehabilitation. Cancer 2001;92(4 Suppl):970-4.

17. Cheville A. Rehabilitation of patients with advanced cancer. Cancer 2001;92(4 Suppl):1039-48.

18. Jung HY. Impact and application of the internal classification functioning, disability and health in the medical rehabilitation. J Korean Acad Rehabil Med 2004;28:401-11.

19. Abrahm JL, Banffy MB, Harris MB. Spinal cord compression in patients with advanced metastatic cancer: "all I care about is walking and living my life". JAMA 2008;299:937-46.

20. Stubblefield MD, Keole N. Upper body pain and functional disorders in patients with breast cancer. PM R 2014;6:170-83.

21. Stubblefield MD, Custodio CM. Upper-extremity pain disorders in breast cancer. Arch Phys Med Rehabil 2006;87(3 Suppl 1):S96-9.

22. Gheita TA, Ezzat Y, Sayed S, El-Mardenly G, Hammam W. Musculoskeletal manifestations in patients with malignant disease. Clin Rheumatol 2010;29:181-8.

23. Yang EJ, Chung SH, Jeon JY, Seo KS, Shin HI, Hwang $\mathrm{JH}$, et al. current practice and barriers in cancer rehabilitation: perspectives of Korean physiatrists. Cancer Res Treat 2015;47:370-8. 